\title{
Utilization of Prostate Cancer Screening According to Dietary Patterns and Other Demographic Variables. The Adventist Health Study-2
}

\author{
Yermek Ibrayev, Keiji Oda, Gary E Fraser, Synnove F Knutsen ${ }^{\bowtie}$ \\ Department of Epidemiology, Biostatistics, and Population Medicine, Loma Linda University, Loma Linda, CA 92350, USA. \\ $\square$ Corresponding author: Synnove Knutsen, MD, PhD, Professor and Chair, Department of Epidemiology, Biostatistics, and Population \\ Medicine, School of Public Health and Professor of Preventive Medicine, School of Medicine; 24951 North Circle Drive, Nichol Hall 2003B, \\ Loma Linda University, Loma Linda, CA 92350, USA. Tel: 909-558-4300 ext. 44590; Fax: +1 909558 0326; E-mail: sknutsen@llu.edu. \\ (C) Ivyspring International Publisher. This is an open-access article distributed under the terms of the Creative Commons License (http://creativecommons.org/ \\ licenses/by-nc-nd/3.0/). Reproduction is permitted for personal, noncommercial use, provided that the article is in whole, unmodified, and properly cited.
}

Received: 2013.04.10; Accepted: 2013.06.05; Published: 2013.06.28

\begin{abstract}
Background: Prostate-specific antigen test and digital rectal examination are considered important screening methods for early detection of prostate cancer. However, the utilization of prostate cancer screening varies widely and there is limited knowledge of the predictors of utilization.

Methods: Self-reported prostate cancer screening utilization within the last 2 years was investigated among II,162 black and non-black North American Seventh-day Adventist men, aged 50-75 years, with different dietary patterns and lifestyle characteristics.

Results: Blacks were more likely to screen for prostate cancer than non-blacks (Odds Ratio $(\mathrm{OR})=1.38$ (95\% confidence interval (Cl): I.20-1.57).

Those with a vegetarian diet, especially vegans, were less likely to follow screening guidelines, particularly among non-Blacks: vegans $(O R=0.47,0.39-0.58)$, lacto-ovo-vegetarians $(O R=0.75$, $0.66-0.86)$, and pesco-vegetarians $(\mathrm{OR}=0.74,0.60-0.91)$ compared to non-vegetarians after adjusting for age, BMI, marital status, education, income, and family history of cancer. Trends for dietary patterns remained unchanged after stratification on age, family history of cancer, education, personal income, marital status, and BMI.

Among black men, diet patterns showed no significant associations with utilization of prostate cancer screening, although vegans tended to underutilize screening compared to non-vegetarians $(\mathrm{OR}=0.70,0.44-1.10)$.

Conclusions: Vegetarians, especially non-black vegans, are less likely to follow recommended prostate cancer screening guidelines. The effect of diet was attenuated, and not statistically significant, among black men.

Impact: Since only about $60 \%$ of US men follow prostate cancer screening guidelines, it is important to study reasons for non-compliance in order to increase utilization of preventive measures against prostate cancer.
\end{abstract}

Key words: PSA, digital rectal exam, prostate cancer screening, lifestyle predictors, dietary pattern, vegetarian, vegan, black men.

\section{Introduction}

Prostate cancer is the most common incident cancer and the second leading cause of cancer deaths among men of all races in the Unites States [1], with 1 in 6 American men developing the disease (lifetime risk $17.18 \%$ ) while lifetime risk of dying of prostate cancer is $2.75 \%[2,3]$. Prostate cancer screening, de- 
spite some acknowledged limitations, is considered an important method for early detection [4, 5]. Screening recommendations in 2002, at the time of recruitment to the Adventist Health Study 2 (AHS-2), were annual or biennial prostate specific antigen (PSA) test and digital rectal examination (DRE) among men 50 years and older with a remaining life expectancy of 10 years or more $[6,7]$.

Following the recommendations for PSA screening frequency has been decreasing from 20.6\% in 2000 to $15.2 \%$ in 2005 among single, never-married men, while screening among married men increased from $29.9 \%$ to $32.6 \%$ in the same time period [8]. At the same time, national trends in age-adjusted prostate cancer mortality in the U.S. declined from 39.31 per 100,000 in 1991 to 21.99 in 2009 [9]. The lower mortality is most likely due to earlier diagnosis and more effective treatment among those who undergo screening [10-15].

Previous research in the U.S. [16, 17] has found that more than $50 \%$ of men, aged 50 and older, report that they have had a PSA test at least once. Utilization of PSA screening has been associated with age, education, health behavior, health insurance, usual source of medical care, and providers' recommendations [18-21]. One study reported that those with a low fat diet and high intake of fruits and vegetables were more likely to use PSA screening [22].

The Adventist church encourages members to abstain from alcohol and tobacco, to adhere to a vegetarian diet and in general follow a healthy lifestyle. Adherence to the dietary recommendations varies widely and thus provides good power to study associations with various dietary patterns. Due to their overall more healthy lifestyle, Adventists enjoy a lower incidence of many chronic diseases compared to the general population [23]. It is of interest to investigate whether this could be related to better screening utilization and whether such utilization varies with self-selected dietary habits as well as with other demographic factors that may confound this association. The recently established cohort of Adventists, the AHS-2, was used for this study [24].

\section{Materials and methods}

\section{Study population}

The Adventist Health Study-2 is a cohort study of 96,335 Adventists living in the U.S. and Canada. The goal of the study is to investigate how diet and other lifestyle factors are associated with colon, prostate and breast cancer. The study has been described in detail elsewhere [24]. Briefly, the AHS-2 is comprised of subjects (30+ years of age) from Adventist churches throughout all 50 U.S. states as well as all
Canadian provinces. Subjects who were sufficiently fluent in English to complete a lengthy questionnaire were eligible for enrollment. Recruitment was from 2002-2007 and included 7,390 black and 25,572 non-black men. The present study is limited to males 50-74 years at baseline, with no prevalent prostate cancer and with complete information on the question of prostate cancer screening. Thus, the final analytic population consists of 2,366 blacks and 8,796 non-blacks (including 7,723 Non-Hispanic Whites, 422 Hispanic and 651 men from other ethnicities), for a total of 11,162 men.

\section{Outcome measure}

The baseline questionnaire asked 2 questions about prostate cancer screening: "How long has it been since you've had ... a PSA blood test?" and "...a digital rectal exam for enlarged prostate (the physician places a gloved finger in the rectum)?", with answering alternatives "never", "5 years or more", "3-4 years ago" and "0-2 years ago". Those who responded that they had been screened with a PSA blood test and/or by DRE within the last 2 years were considered compliant according to the recommendations of the American Cancer Society (ACS). This "Yes/No" prostate cancer screening measure was the dependent variable in our regression analyses.

Dietary Pattern classification was based on the intake of 25 different food group items [25]. Participants were asked to report their usual or average intake of each food item during the past one year. There were up to 9 frequency response options, ranging from "never or rarely" to " $6+$ per day". Frequencies of intake were converted to daily equivalents and these were used to construct composite food variables that measured intake of red meat, poultry, fish, eggs and dairy foods. Values of these variables allowed subjects to be assigned to one of five different dietary groups as shown below:

- Vegans: eat meat, fish, and dairy less than once a month;

- Lacto/ovo-vegetarians: eat meat and/or fish less than once a month, and dairy and/or eggs more than once a month;

- Pesco-vegetarians: eat meat less than once a month and fish more than once a month;

- Semi-vegetarians: eat meat more than once a month, and fish and meat combined less than once a week;

- Non-vegetarians: eat meat and fish combined 1 or more times per week, and meat $>1 /$ month.

Demographic variables examined were based on literature review and included age, race/ethnicity, personal annual income, educational attainment, 
marital status and geographic region of residence [26, 27].

Family history of cancer was registered in two ways in the questionnaire. For prostate cancer, the respondents were asked to indicate whether first-degree relatives (father, brothers or sons) had been diagnosed with this cancer. In addition there was another field where subjects could tick and write-in which other cancers had occurred among first-degree relatives. Sometimes the men wrote in prostate cancer in this field and, if so, these were added to the analytic file as prostate cancer in the former variable. Thus, we defined four risk groups depending on whether there was a family history of prostate cancer only $(\mathrm{N}=581)$, other cancer only $(\mathrm{N}=3,685)$, both prostate and other cancers $(\mathrm{N}=522)$ or no cancer $(\mathrm{N}=5,438)$.

Race was self-reported and subjects could choose among a total of 22 different race/ethnicities. Anyone indicating a mixture of several races where "black" was one component was categorized as black. Likewise, subjects were categorized as Hispanic if there was any mention of "Hispanic" in their racial description. Race was a confounder since prostate cancer is known to be more common among black men and thus screening utilization could also be affected by the higher occurrence of prostate cancer in this racial group.

\section{Other candidate covariables}

Body mass index was calculated from self-reported height and weight.

Physical activity was estimated based on self-reported time of a vigorous exercise program in minutes per week and/or distance and time spent in walking, running or jogging each week. Based on these questions, physical exercise was categorized into 4 levels: none, low, medium and high. For example, medium exercise required either walking, running or jogging for between 105 to 175 minutes per week or for a distance between 3 to 9 miles per week. A vigorous exercise program was defined as vigorous exercise more than 105 minutes per week.

Alcohol consumption - Since Adventists are mostly teetotalers, alcohol consumption was classified as Never vs. Ever use of alcohol.

Smoking - as with alcohol, the Adventist church proscribes smoking. Only 1.3\% report being current smokers and $71.7 \%$ of the men have never smoked. The smoking variable was therefore dichotomized into Never versus Ever.

Prevalent chronic diseases - at enrollment, subjects were asked about prevalence of a total of 30 doctor-diagnosed chronic diseases including respiratory, cardiac, endocrine, neurologic and gastrointestinal diseases. Based on this, "Number of chronic diseases" was coded into three levels: "none", "1-4" and "5 or more". In addition, "Enlarged prostate" was reported as a separate variable.

\section{Statistical analyses}

Chi square statistics were used to test univariate null hypotheses that prostate screening was not related to other nominated variables, in both the total sample and samples stratified by race (table 1 ).

Age-adjusted univariate logistic regression analysis was used to explore the independent association between prostate cancer screening behavior and dietary patterns. Based on the literature [18-20] a basic model that included dietary patterns, age, race, education and marital status was developed. Using this basic model, other candidate variables listed in Table 1 were added one at a time to assess whether they changed the main effect of dietary patterns more than $10 \%$. Only BMI met this criterion and was included in the final model (table 2).

Interactions between race (black and non-black) and the dietary patterns were found and therefore the analyses were performed separately by race (table 2). Development of final models for the two racial groups used the same approach as described above and the final models for both blacks and non-blacks were identical to the model developed for the total study population.

Calculation of variance inflation factors did not reveal excessive multicollinearity in these models. All analyses were done using SAS Software 9.3 [28].

\section{Results}

About three fourths of the men reported having been screened for prostate cancer during the last 2 years. And among those who had not been screened within the last 2 years, one third $(n=951)$ had never undergone prostate cancer screening. A larger proportion of black than non-black men reported screening within the last 2 years $(75.0 \%$ vs. $72.8 \%$, respectively). In multivariate analysis the odds ratio (OR) was larger than that implied by this univariate result with a value of 1.38 (95\% confidence interval (CI): 1.20-1.57).

Additionally, those who reported screening according to ACS guidelines were more likely to be older, to be currently married, to have higher BMI, education and income, be more physically active and report a higher number of prevalent chronic diseases (Table 1).

\section{Association with dietary patterns}

A higher than expected proportion of those who had not screened according to ACS guidelines tended 
to be vegans (13.3 vs. $6.8 \%$ ), and the overall association between screening within two years and dietary pattern was statistically significant $(p<0.0001)$ (Table $1)$.

In age-adjusted univariate analyses, those with vegetarian dietary patterns were less likely to have utilized prostate cancer screening than non-vegetarians. The vegans were least likely to screen $(\mathrm{OR}=0.46)$ followed by the lacto-ovo-vegetarians $(\mathrm{OR}=0.84)$ and pesco-vegetarians $(\mathrm{OR}=0.88)$. No difference in screening utilization patterns was found between semi-vegetarians and non-vegetarians (Table 2).

When adjusting for a number of demographic factors in multivariable analyses, the association with dietary patterns remained very similar for vegans, lacto-ovo-vegatarians and pesco-vegetarians with OR $0.50,0.76$ and 0.79 , respectively (Table 2 ).

\section{Race specific analyses}

The association with dietary patterns was much stronger among non-blacks than blacks with the multivariate odds ratio $(95 \% \quad \mathrm{CI})$ comparing to non-vegetarians of $0.47(0.39-0.58)$ for vegans, $0.75(0.66-0.86)$ for lacto-ovo- and $0.74(0.60-0.91)$ for pesco-vegetarians. The weaker associations with diet among black subjects did include a point estimate of 0.70 (0.44-1.10) for vegans compared to non-vegetarians, and odds ratios for other vegetarian groups that were all less than 1.0, although not statistically significant (Table 2).
Notice (Table 2) that higher BMI is independently and significantly $(\mathrm{p}<0.001)$ related to screening utilization, and is also very strongly related to vegetarian status [29]. Thus BMI potentially confounds the diet-screening association, and for this reason all analyses in Table 2 are adjusted for BMI. For comparison, when there is no adjustment for BMI, multivariate odds ratios ( $95 \%$ confidence intervals) for all subjects combined are $0.43 \quad(0.37-0.51), 0.71$ (0.63-0.80), 0.75 (0.63-0.89), and 1.05 (0.82-1.34) for vegans, lacto-ovo-, pesco-, and semi-vegetarians, when compared to non-vegetarians. These are stronger associations, which raise the possibility that BMI may be an intermediary between diet and screening. Specifically, vegetarians have lower BMI, and this is associated with less screening activity. In black subjects, similar statistics not adjusted for BMI are $0.61(0.39-0.94), 0.78(0.56-1.08), 0.89(0.63-1.27)$, and $0.64(0.35-1.20)$.

\section{Stratified analyses}

The lower screening utilization among non-black vegetarians, and especially among vegans, remained virtually unchanged when stratifying on a number of demographic variables including age, education, marital status, BMI, income and family history of cancer (Table 3). Similarly, the attenuated association between dietary patterns and screening behavior found among black men remained virtually unchanged when stratifying on these same demographic variables.

Table I. Demographic characteristics of the analytic population according to prostate screening status*.

\begin{tabular}{|c|c|c|c|c|c|c|}
\hline \multirow[t]{2}{*}{ Variables } & \multicolumn{2}{|c|}{ Total N=11,162 (100.0\%) } & \multicolumn{2}{|c|}{ Blacks $N=2,366(21.2 \%)$} & \multicolumn{2}{|c|}{ Non-blacks N=8,796 (78.8\%) } \\
\hline & $\begin{array}{l}\text { Screened } \\
(\mathrm{n}=8178,73.3 \%)\end{array}$ & $\begin{array}{l}\text { Not screened } \\
(\mathrm{n}=2984,26.7 \%)\end{array}$ & $\begin{array}{l}\text { Screened } \\
(\mathrm{n}=1773,75.0 \%)\end{array}$ & $\begin{array}{l}\text { Not screened } \\
(n=593,25.0 \%)\end{array}$ & $\begin{array}{l}\text { Screened } \\
(\mathrm{n}=6405,72.8 \%)\end{array}$ & $\begin{array}{l}\text { Not screened } \\
(\mathrm{n}=2391,27.2 \%)\end{array}$ \\
\hline Mean age (SD) & $61.7(7.0)$ & $59.5(6.8)$ & $60.2(6.8)$ & $59.2(6.8)$ & $62.1(7.0)$ & $59.6(6.8)$ \\
\hline \multirow[t]{2}{*}{ p-value } & $<.0001$ & & $<.001$ & & $<.0001$ & \\
\hline & $\%$ & $\%$ & $\%$ & $\%$ & $\%$ & $\%$ \\
\hline \multicolumn{7}{|l|}{ Age, years } \\
\hline $50-59$ & 48.3 & 61.0 & 58.9 & 66.4 & 45.7 & 59.9 \\
\hline $60-69$ & 38.4 & 30.5 & 31.9 & 26.9 & 40.0 & 31.3 \\
\hline $70-74$ & 13.3 & 8.4 & 9.2 & 6.7 & 14.3 & 8.8 \\
\hline p-value & $<.0001$ & & 0.0045 & & $<.0001$ & \\
\hline \multicolumn{7}{|l|}{ Dietary status } \\
\hline Vegan & 6.8 & 13.3 & 6.2 & 9.1 & 7.0 & 14.2 \\
\hline Lacto-ovo-vegetarian & 29.2 & 30.6 & 16.0 & 16.5 & 32.4 & 33.5 \\
\hline Pesco-vegetarian & 9.2 & 8.9 & 14.1 & 12.5 & 8.0 & 8.1 \\
\hline Semi-vegetarian & 5.2 & 3.9 & 2.8 & 3.7 & 5.8 & 3.9 \\
\hline Non-vegetarian & 49.6 & 43.4 & 61.0 & 58.1 & 46.8 & 40.3 \\
\hline $\mathrm{p}$-value & $<.0001$ & & 0.3078 & & $<.0001$ & \\
\hline \multicolumn{7}{|l|}{ Race } \\
\hline Black & 19.8 & 17.29 & 100.0 & 100.0 & . & . \\
\hline Non-black & 80.2 & 82.71 & . & . & 100.0 & 100.0 \\
\hline p-value & 0.0387 & & & & & \\
\hline
\end{tabular}




\begin{tabular}{|c|c|c|c|c|c|c|}
\hline \multirow[t]{2}{*}{ Variables } & \multicolumn{2}{|c|}{ Total N=11,162 (100.0\%) } & \multicolumn{2}{|c|}{ Blacks N=2,366 (21.2\%) } & \multicolumn{2}{|c|}{ Non-blacks N=8,796 (78.8\%) } \\
\hline & $\begin{array}{l}\text { Screened } \\
(\mathrm{n}=8178,73.3 \%)\end{array}$ & $\begin{array}{l}\text { Not screened } \\
(\mathrm{n}=2984,26.7 \%)\end{array}$ & $\begin{array}{l}\text { Screened } \\
(\mathrm{n}=1773,75.0 \%)\end{array}$ & $\begin{array}{l}\text { Not screened } \\
(\mathrm{n}=593,25.0 \%)\end{array}$ & $\begin{array}{l}\text { Screened } \\
(\mathrm{n}=6405,72.8 \%)\end{array}$ & $\begin{array}{l}\text { Not screened } \\
(\mathrm{n}=2391,27.2 \%)\end{array}$ \\
\hline Never married & 1.9 & 4.0 & 2.7 & 4.8 & 1.7 & 3.9 \\
\hline Married & 90.5 & 84.6 & 87.4 & 81.9 & 91.3 & 85.1 \\
\hline Divorced & 7.6 & 11.4 & 10.0 & 13.3 & 7.0 & 11.0 \\
\hline p-value & $<.0001$ & & 0.0002 & & $<.0001$ & \\
\hline \multicolumn{7}{|l|}{ Education } \\
\hline College or less & 38.3 & 47.5 & 44.4 & 55.2 & 36.8 & 45.9 \\
\hline Bachelor's degree & 30.2 & 29.3 & 28.5 & 23.5 & 30.6 & 30.6 \\
\hline Graduate degree & 31.5 & 23.1 & 27.2 & 21.3 & 32.6 & 23.5 \\
\hline p-value & $<.0001$ & & $<.0001$ & & $<.0001$ & \\
\hline \multicolumn{7}{|c|}{ Annual personal income } \\
\hline$<\$ 20 K$ & 19.2 & 28.1 & 22.1 & 32.8 & 18.5 & 27.2 \\
\hline$\$ 21 K-50 K$ & 44.2 & 44.5 & 45.0 & 49.3 & 44.0 & 43.5 \\
\hline$\$ 51 \mathrm{~K}-74 \mathrm{~K}$ & 18.6 & 12.7 & 20.2 & 8.5 & 18.2 & 13.6 \\
\hline$\geq \$ 75 \mathrm{~K}$ & 18.1 & 14.6 & 12.7 & 9.3 & 19.4 & 15.7 \\
\hline $\mathrm{p}$-value & $<.0001$ & & $<.0001$ & & $<.0001$ & \\
\hline \multicolumn{7}{|c|}{ Body mass index, $\mathrm{kg} / \mathrm{m}^{2}$} \\
\hline $16-19.9$ & 1.9 & 4.3 & 2.0 & 3.2 & 1.9 & 4.6 \\
\hline $20-24.9$ & 29.2 & 34.0 & 24.0 & 28.5 & 30.5 & 35.2 \\
\hline $25-29.9$ & 46.1 & 42.6 & 47.9 & 46.9 & 45.7 & 41.6 \\
\hline $30+$ & 22.7 & 19.1 & 26.1 & 21.3 & 21.9 & 18.6 \\
\hline p-value & $<.0001$ & & 0.0092 & & $<.0001$ & \\
\hline \multicolumn{7}{|l|}{ Smoking } \\
\hline Never & 71.9 & 71.3 & 66.8 & 65.3 & 73.2 & 72.5 \\
\hline Ever & 28.1 & 28.7 & 33.2 & 34.7 & 26.8 & 27.5 \\
\hline p-value & 0.2062 & & 0.3781 & & 0.2719 & \\
\hline \multicolumn{7}{|l|}{ Alcohol use } \\
\hline Never & 52.2 & 49.2 & 43.8 & 40.0 & 54.3 & 51.2 \\
\hline Ever & 47.8 & 50.8 & 56.2 & 60.0 & 45.7 & 48.8 \\
\hline $\mathrm{p}$-value & 0.0002 & & 0.0409 & & 0.0007 & \\
\hline \multicolumn{7}{|l|}{ Physical exercise } \\
\hline Never & 20.3 & 25.3 & 25.9 & 31.2 & 18.9 & 24.0 \\
\hline Low level & 21.4 & 23.1 & 21.3 & 24.3 & 21.4 & 22.9 \\
\hline Middle level & 35.1 & 32.0 & 30.9 & 25.9 & 36.2 & 33.3 \\
\hline High/Very high level & 23.2 & 19.6 & 21.9 & 18.7 & 23.6 & 19.8 \\
\hline $\mathrm{p}$-value & $<.0001$ & & 0.0025 & & $<.0001$ & \\
\hline \multicolumn{7}{|l|}{ Family history of cancer } \\
\hline No cancer & 51.5 & 58.0 & 60.2 & 69.5 & 49.2 & 55.4 \\
\hline Prostate cancer only & 6.2 & 4.3 & 7.3 & 3.7 & 5.8 & 4.5 \\
\hline Other cancers only & 36.9 & 33.4 & 28.6 & 23.4 & 39.2 & 35.7 \\
\hline $\begin{array}{l}\text { Both prostate and other } \\
\text { cancers }\end{array}$ & 5.4 & 4.3 & 4.0 & 3.5 & 5.8 & 4.5 \\
\hline $\mathrm{p}$-value & $<.0001$ & & 0.0003 & & $<.0001$ & \\
\hline \multicolumn{7}{|l|}{$\begin{array}{l}\text { Number of chronic dis- } \\
\text { eases }\end{array}$} \\
\hline 0 & 23.9 & 45.6 & 23.4 & 40.5 & 24.0 & 46.6 \\
\hline $1-4$ & 70.0 & 51.7 & 71.7 & 55.7 & 69.5 & 50.9 \\
\hline $5+$ & 6.1 & 2.7 & 5.0 & 3.7 & 6.4 & 2.5 \\
\hline $\mathrm{p}$-value & $<.0001$ & & $<.0001$ & & $<.0001$ & \\
\hline \multicolumn{7}{|l|}{ Enlarged prostate } \\
\hline Never diagnosed & 78.9 & 91.9 & 84.7 & 91.5 & 77.5 & 92.0 \\
\hline 0-4 yrs ago diagnosed & 11.0 & 2.5 & 10.5 & 3.2 & 11.1 & 2.3 \\
\hline $5+$ yrs ago diagnosed & 10.1 & 5.6 & 4.9 & 5.3 & 11.4 & 5.6 \\
\hline p-value & $<.0001$ & & $<.0001$ & & $<.0001$ & \\
\hline
\end{tabular}

* Screening refers to screening within the previous two years. $\dagger$ p-value refers to tests of null hypotheses of no association between prostate screening and categories of the listed variables. 
Table 2. Odds ratios for prostate cancer screening test utilization within last 2 years. Total and by race.

\begin{tabular}{|c|c|c|c|c|c|c|}
\hline \multirow[t]{3}{*}{ Variables } & \multicolumn{2}{|l|}{ Total } & \multicolumn{2}{|l|}{ Blacks } & \multicolumn{2}{|l|}{ Non-blacks } \\
\hline & $\begin{array}{l}\text { Age-adjusted model } \\
(\mathrm{N}=9451)\end{array}$ & $\begin{array}{l}\text { Multivariable model } \\
(\mathrm{N}=8555)\end{array}$ & $\begin{array}{l}\text { Age-adjusted } \\
\text { model }(\mathrm{N}=1863)\end{array}$ & $\begin{array}{l}\text { Multivariable model } \\
(\mathrm{N}=1649)\end{array}$ & $\begin{array}{l}\text { Age-adjusted } \\
\text { model }(\mathrm{N}=7588)\end{array}$ & $\begin{array}{l}\text { Multivariable } \\
\text { model }(\mathrm{N}=6906)\end{array}$ \\
\hline & $\mathrm{OR}(95 \% \mathrm{CI}) \dagger$ & $\mathrm{OR}(95 \% \mathrm{CI}) \ddagger$ & OR $(95 \% \mathrm{CI}) \dagger$ & $\mathrm{OR}(95 \% \mathrm{Cl}) \ddagger$ & OR $(95 \% \mathrm{Cl}) \dagger$ & $\mathrm{OR}(95 \% \mathrm{CI}) \ddagger$ \\
\hline \multicolumn{7}{|l|}{ Dietary pattern } \\
\hline Vegan & $0.46(0.40-0.54)$ & $0.50(0.42-0.60)$ & $0.70(0.47-1.05)$ & $0.70(0.44-1.10)$ & $0.44(0.37-0.52)$ & $0.47(0.39-0.58)$ \\
\hline Lacto-ovo-vegetarian & $0.84(0.75-0.94)$ & $0.76(0.67-0.86)$ & $1.02(0.75-1.38)$ & $0.79(0.56-1.10)$ & $0.83(0.74-0.94)$ & $0.75(0.66-0.86)$ \\
\hline Pesco-vegetarian & $0.88(0.75-1.04)$ & $0.79(0.66-0.95)$ & $1.07(0.78-1.49)$ & $0.96(0.67-1.37)$ & $0.81(0.67-0.98)$ & $0.74(0.60-0.91)$ \\
\hline Semi-vegetarian & $1.15(0.91-1.46)$ & $1.11(0.86-1.43)$ & $0.71(0.39-1.28)$ & $0.74(0.38-1.43)$ & $1.27(0.98-1.64)$ & $1.17(0.89-1.54)$ \\
\hline Non-vegetarian & Reference $* * *$ & Reference $^{* \star *}$ & Reference & Reference & Reference ${ }^{* * *}$ & Reference $^{* \star *}$ \\
\hline \multicolumn{7}{|l|}{ Age, years } \\
\hline $50-54$ & Reference $* * *$ & Reference $* * *$ & Reference $^{* * *}$ & Reference ${ }^{* * *}$ & Reference ${ }^{* * *}$ & Reference $^{* \star *}$ \\
\hline $55-59$ & $1.40(1.23-1.58)$ & $1.47(1.29-1.68)$ & $1.21(0.93-1.59)$ & $1.24(0.93-1.67)$ & $1.46(1.27-1.68)$ & $1.54(1.33-1.79)$ \\
\hline $60-64$ & $1.61(1.41-1.83)$ & $1.87(1.62-2.16)$ & $1.46(1.06-2.00)$ & $1.76(1.24-2.51)$ & $1.68(1.45-1.95)$ & $1.92(1.64-2.25)$ \\
\hline $65-69$ & $2.23(1.92-2.59)$ & $2.71(2.30-3.19)$ & $1.61(1.13-2.28)$ & $1.82(1.24-2.67)$ & $2.45(2.07-2.89)$ & $2.96(2.47-3.54)$ \\
\hline $70-74$ & $2.34(1.98-2.76)$ & $3.11(2.58-3.75)$ & $1.67(1.10-2.53)$ & $2.08(1.30-3.34)$ & $2.56(2.14-3.07)$ & $3.38(2.75-4.14)$ \\
\hline \multicolumn{7}{|l|}{ Race } \\
\hline Black vs. Non-black & $1.21(1.07-1.37)$ & $1.38(1.20-1.57)$ & & & & \\
\hline \multicolumn{7}{|l|}{ Body mass index, $\mathrm{kg} / \mathrm{m}^{2}$} \\
\hline $16-19.9$ & $0.62(0.47-0.82)$ & $0.64(0.48-0.86)$ & $0.75(0.38-1.49)$ & $0.84(0.39-1.78)$ & $0.59(0.43-0.81)$ & $0.62(0.44-0.85)$ \\
\hline $20-24.9$ & Reference $^{* \star *}$ & Reference $^{* \star *}$ & Reference* & Reference* & Reference ${ }^{* \star *}$ & Reference $^{* * *}$ \\
\hline $25-29.9$ & $1.21(1.08-1.35)$ & $1.19(1.06-1.34)$ & $1.23(0.95-1.61)$ & $1.18(0.89-1.56)$ & $1.19(1.05-1.34)$ & $1.19(1.05-1.36)$ \\
\hline$\geq 30$ & $1.32(1.15-1.51)$ & $1.39(1.20-1.61)$ & $1.41(1.03-1.94)$ & $1.38(0.99-1.94)$ & $1.28(1.10-1.49)$ & $1.39(1.18-1.64)$ \\
\hline \multicolumn{7}{|l|}{ Education } \\
\hline College or less & $0.69(0.62-0.77)$ & $0.74(0.66-0.83)$ & $0.67(0.52-0.88)$ & $0.73(0.55-0.98)$ & $0.68(0.60-0.77)$ & $0.73(0.64-0.84)$ \\
\hline Bachelor's degree & Reference $^{* * *}$ & Reference $^{* * *}$ & Reference $^{\star * *}$ & Reference* $^{*}$ & Reference $^{* \star *}$ & Reference $^{* * *}$ \\
\hline Postgraduate degree & $1.39(1.22-1.59)$ & $1.25(1.09-1.44)$ & $1.21(0.88-1.67)$ & $0.96(0.67-1.37)$ & $1.42(1.23-1.64)$ & $1.30(1.12-1.52)$ \\
\hline \multicolumn{7}{|l|}{ Annual personal income } \\
\hline$<\$ 20 \mathrm{~K}$ & $0.62(0.55-0.69)$ & $0.70(0.61-0.79)$ & $0.70(0.54-0.90)$ & $0.80(0.61-1.05)$ & $0.59(0.52-0.67)$ & $0.67(0.58-0.77)$ \\
\hline$\$ 21 \mathrm{~K}-50 \mathrm{~K}$ & Reference $* * *$ & Reference $* * *$ & Reference $* * *$ & Reference ${ }^{* \star *}$ & Reference ${ }^{* \star *}$ & Reference $^{* * *}$ \\
\hline$\$ 51 \mathrm{~K}-74 \mathrm{~K}$ & $1.67(1.44-1.93)$ & $1.54(1.32-1.80)$ & $2.69(1.83-3.96)$ & $2.64(1.75-3.98)$ & $1.53(1.30-1.79)$ & $1.39(1.18-1.65)$ \\
\hline$\geq \$ 75 \mathrm{~K}$ & $1.45(1.26-1.67)$ & $1.25(1.07-1.45)$ & $1.67(1.12-2.47)$ & $1.43(0.94-2.20)$ & $1.45(1.24-1.69)$ & $1.20(1.02-1.42)$ \\
\hline \multicolumn{7}{|l|}{ Marital status } \\
\hline Non-married vs. Married & $0.56(0.49-0.65)$ & $0.65(0.56-0.76)$ & $0.67(0.50-0.90)$ & $0.69(0.50-0.96)$ & $0.53(0.45-0.62)$ & $0.64(0.54-0.76)$ \\
\hline \multicolumn{7}{|l|}{ Family history of cancer } \\
\hline No cancer & Reference ${ }^{\star \star *}$ & Reference $^{\star \star \star}$ & Reference $^{\star *}$ & Reference $^{*}$ & Reference ${ }^{* \star *}$ & Reference $^{* \star *}$ \\
\hline Prostate cancer only & $1.58(1.27-1.97)$ & $1.59(1.26-2.01)$ & $2.34(1.35-4.05)$ & $1.99(1.11-3.57)$ & $1.48(1.16-1.88)$ & $1.53(1.18-1.98)$ \\
\hline Other cancer only & $1.24(1.12-1.37)$ & $1.22(1.09-1.35)$ & $1.41(1.10-1.81)$ & $1.35(1.03-1.76)$ & $1.23(1.10-1.37)$ & $1.19(1.06-1.34)$ \\
\hline $\begin{array}{l}\text { Both prostate and other } \\
\text { cancer }\end{array}$ & $1.35(1.08-1.69)$ & $1.19(0.93-1.51)$ & $1.42(0.79-2.56)$ & $1.11(0.59-2.09)$ & $1.36(1.06-1.73)$ & $1.21(0.93-1.57)$ \\
\hline
\end{tabular}


Table 3. Odds ratios ( $95 \%$ confidence intervals) for PSA and/or DRE-test utilization within last 2 years by dietary pattern: Possible effect modification by other selected variables.

\begin{tabular}{|c|c|c|}
\hline Dietary pattern & \multicolumn{2}{|c|}{ All subjects, OR $(95 \% \mathrm{CI}) \dagger$} \\
\hline Vegan & $0.50(0.81-0.58)$ & \\
\hline Lacto-ovo-vegetarian & $0.81(0.73-0.90)$ & \\
\hline Pesco-vegetarian & $0.80(0.68-0.93)$ & \\
\hline Semi-vegetarian & $1.18(0.95-1.47)$ & \\
\hline Non-vegetarian & Reference $^{* * *}$ & \\
\hline Stratification variables & $\begin{array}{l}1^{\text {st }} \text { strata, OR }(95 \% \\
\text { CI) }\end{array}$ & $\begin{array}{l}2^{\text {nd }} \text { strata, OR }(95 \% \\
\text { CI) }\end{array}$ \\
\hline by age & $50-59$ years old & $60-74$ years old \\
\hline Vegan & $0.53(0.43-0.65)$ & $0.47(0.37-0.58)$ \\
\hline Lacto-ovo-vegetarian & $0.80(0.70-0.92)$ & $0.82(0.69-0.96)$ \\
\hline Pesco-vegetarian & $0.86(0.69-1.06)$ & $0.73(0.59-0.92)$ \\
\hline Semi-vegetarian & $1.17(0.87-1.57)$ & $1.19(0.85-1.65)$ \\
\hline Non-vegetarian & Reference ${ }^{* * *}$ & Reference $^{* * *}$ \\
\hline by race & Black & Non-black \\
\hline Vegan & $0.77(0.53-1.13)$ & $0.46(0.39-0.54)$ \\
\hline Lacto-ovo-vegetarian & $0.88(0.65-1.19)$ & $0.79(0.70-0.89)$ \\
\hline Pesco-vegetarian & $0.99(0.73-1.35)$ & $0.74(0.62-0.88)$ \\
\hline Semi-vegetarian & $0.78(0.43-1.40)$ & $1.23(0.97-1.56)$ \\
\hline Non-vegetarian & Reference & Reference ${ }^{* * *}$ \\
\hline by education level & $\begin{array}{l}\text { Less than bachelors' } \\
\text { level }\end{array}$ & $\begin{array}{l}\text { Bachelors' and } \\
\text { higher degree }\end{array}$ \\
\hline Vegan & $0.54(0.44-0.66)$ & $0.47(0.37-0.58)$ \\
\hline Lacto-ovo-vegetarian & $0.77(0.66-0.90)$ & $0.85(0.72-0.99)$ \\
\hline Pesco-vegetarian & $0.74(0.60-0.90)$ & $0.89(0.70-1.13)$ \\
\hline Semi-vegetarian & $1.10(0.83-1.47)$ & $1.30(0.92-1.85)$ \\
\hline Non-vegetarian & Reference ${ }^{* * *}$ & Reference $^{* * *}$ \\
\hline by marital status & Non-married & Married \\
\hline Vegan & $0.55(0.36-0.85)$ & $0.49(0.42-0.58)$ \\
\hline Lacto-ovo-vegetarian & $0.80(0.58-1.10)$ & $0.81(0.72-0.91)$ \\
\hline Pesco-vegetarian & $0.67(0.43-1.05)$ & $0.81(0.69-0.96)$ \\
\hline Semi-vegetarian & $1.37(0.71-2.65)$ & $1.16(0.92-1.46)$ \\
\hline Non-vegetarian & Reference $^{* *}$ & Reference $^{* * *}$ \\
\hline by BMI category & $<25$ & $\geq 25.0$ \\
\hline Vegan & $0.45(0.36-0.55)$ & $0.57(0.45-0.72)$ \\
\hline Lacto-ovo-vegetarian & $0.79(0.66-0.95)$ & $0.81(0.71-0.92)$ \\
\hline Pesco-vegetarian & $0.64(0.50-0.83)$ & $0.91(0.74-1.11)$ \\
\hline Semi-vegetarian & $1.37(0.91-2.05)$ & $1.09(0.84-1.42)$ \\
\hline Non-vegetarian & Reference ${ }^{* * *}$ & Reference $^{* * *}$ \\
\hline $\begin{array}{l}\text { by personal income } \\
\text { level }\end{array}$ & $<\$ 20 \mathrm{~K}$ & $\geq \$ 20 \mathrm{~K}$ \\
\hline Vegan & $0.48(0.37-0.64)$ & $0.51(0.42-0.61)$ \\
\hline Lacto-ovo-vegetarian & $0.89(0.71-1.11)$ & $0.78(0.69-0.88)$ \\
\hline Pesco-vegetarian & $0.74(0.54-1.01)$ & $0.81(0.68-0.97)$ \\
\hline Semi-vegetarian & $2.12(1.32-3.42)$ & $0.98(0.77-1.26)$ \\
\hline Non-vegetarian & Reference ${ }^{* * *}$ & Reference $^{* * *}$ \\
\hline $\begin{array}{l}\text { by family history of } \\
\text { prostate cancer }\end{array}$ & Yes & No \\
\hline Vegan & $0.50(0.42-0.58)$ & $0.46(0.28-0.75)$ \\
\hline Lacto-ovo-vegetarian & $0.82(0.73-0.91)$ & $0.71(0.50-1.00)$ \\
\hline Pesco-vegetarian & $0.83(0.70-0.97)$ & $0.54(0.33-0.88)$ \\
\hline Semi-vegetarian & $1.18(0.94-1.48)$ & $1.24(0.53-2.86)$ \\
\hline Non-vegetarian & Reference $^{* * *}$ & Reference $^{* *}$ \\
\hline
\end{tabular}

** $\mathrm{p}$-value for trend $<0.01, * * * \mathrm{p}$-value for trend $<0.001$, ${ }^{+}$Model adjusted for age, race, BMI, marital status, education, personal income level, and family history of prostate cancer.

\section{Discussion}

About $74.4 \%$ of respondents reported that they had had either PSA, DRE or both tests, and $66.5 \%$ reported a PSA test performed within the past 2 years. This proportion is higher than that observed in other national samples and surveys during 2000-2003: 51.4-67.4\% of men reported of PSA test utilization in 1996 in a Utah cross-sectional study [19], and 55.2\% reported ever having had a PSA test in the 2003 Health Information National Trends Survey [21]. The larger proportion of screening utilization among black men is in line with that reported by other studies among black men with similar educational background as those in our study [30].

The main demographic predictors of screening that we found (older age, high education, personal income, BMI, married, family history of prostate or other cancers, and number of chronic diseases) were positively associated with recent use of screening services. These findings are also similar to those reported by other studies [17-22, 31]. Lower rates of other cancer screening services such as Pap smears, mammography, and colorectal screening have also been found among those with low income, or uninsured, but in contrast to our rates among black subjects, rates tend to be lower among ethnic minorities [32-40].

Our findings of lower prostate cancer screening utilization among vegetarians and especially among vegans are interesting. Few other studies have reported on the association between screening utilization and diet, but Close et al. [22] reported that subjects with a low fat and high fruit and vegetable diet were significantly more likely to utilize prostate cancer screening $(\mathrm{OR}=2.72, \mathrm{p}<0.05)$. They did not, however, report associations between screening participation and diets varying in meat or dairy intake which is the focus of our study.

The confounding and/or mediation by BMI on the diet-screening association can be illustrated by the vegans who have greatly lower values of BMI on average. As we have also shown that those with lower BMI are less likely to screen, it is no surprise that vegans are less likely to screen. However, independent effects of diet persist when BMI is held constant by adjustment, even though they are generally a little weaker.

The lower screening rates among vegans and lacto-ovo-vegetarians were most marked among non-blacks whereas black males did not show a clear association with dietary patterns. This may be due to the knowledge and understanding among black men that they are at higher risk for prostate cancer than the 
general population. Whether Adventist black males also are at higher risk of prostate cancer has not yet been reported.

Why vegetarians in general and especially vegans do not utilize screening to the same extent as non-vegetarians, needs further exploration. There could be a perception that the risk of prostate cancer or cancer in general is lower among persons eating a vegetarian diet. Although not conclusive, there is some evidence in support of this. Some studies, including a meta-analysis have reported a positive association between red meat, fat intake and prostate cancer [16, 41-43]. Walker et al. [44] report positive associations between prostate cancer risk and consumption of meat and eggs, and negative associations with fruit, vegetables, foods containing more dietary fiber and carotenoids. The previous Adventist Health Study (AHS-1) found that white males eating beans, lentils, peas, tomatoes, and other dried fruits, had a lower risk of prostate cancer during 6 years of follow-up [45], and also that men drinking soy milk at least once a day had significantly lower risk of developing prostate cancer [46]. Wei et al. [47] also found a negative association with tomato products, and others also found an inverse association with soy intake, or the intake of other vegetables and carotenoids [48-51].

Another possible explanation for lower screening rates in vegetarians could be that those who choose a vegetarian diet have a different philosophical approach to life than omnivores, and that this includes lower levels of health care utilization. Findings from California Adventists in the previous AHS-1 have found that, compared to omnivores, vegetarians (mostly lacto-ovo-vegetarians) were less likely to have consulted an MD during the preceding two years, were less likely to have been in the ER and were less likely to have had emergency surgery and emergency X-rays [52]. They were also less likely to have been diagnosed with a number of chronic diseases and allergies. Thus, the lower health care utilization was ascribed to lower prevalence of chronic diseases and thus lower need to utilize the health care system. However, a reverse causal sequence may also be possible. Any tendency to consult physicians less frequently may result in fewer diagnoses of chronic diseases, or that they are diagnosed at a more advanced stage.

A further alternative is that as vegetarian Adventists tend to be better educated $[53,54]$ and likely more health conscious, they would be more aware of both the benefits and limitations/side effects of prostate cancer screening [14,55]. This may have made them more reluctant to undergo screening [56].
These forces would be aggravated if vegetarians tended to have lower health insurance coverage. We have limited information with which to assess this question. A sub-study of 11,000 AHS-2 subjects, the Biopsychosocial Religion and Health Study (BRHS), assessed health care coverage. Preliminary analyses show that the vegan males were less likely to have health insurance coverage than omnivorous men. This may have been by choice, as only the omnivores indicated that they had problems with access to medical care because of costs. Others have found that access to medical care, and providers' recommendations for screening were more important than socioeconomic status and education as determinants of cancer screening utilization $(17,18,20,21,30)$. Whether this applies to U.S. Adventists is not yet clear.

Finally, it is possible that vegetarians, especially vegans, are more religious or have a higher trust in God and thus do not feel the need for screening. In order to test this, we did a sub-analyses among 2,000 men in the BRHS who met the inclusion/exclusion criteria for our study. Frequency of church attendance or the perception of a benevolent God, was no different among vegetarians than among those having a meat-based diet. However, the vegetarians did report spending more time in personal Bible study. When running our final model on this subgroup and including various indicators of religiosity, there was virtually no change in the effect estimates of screening prevalence by dietary patterns. Thus there does not seem to be evidence that the association between prostate cancer screening and dietary patterns is confounded by religiosity.

Adventists emphasize the importance of education and a relatively large proportion of Adventists have health professional degrees. The educational level among vegans is not dramatically different from that of other dietary pattern groups. Thus, it is possible that the decision to not screen for prostate cancer among vegetarians is based on a combination of healthy lifestyle, awareness of the controversies around prostate cancer screening and other unknown factors.

No significant association between diet pattern and screening utilization was found among the blacks although there was a tendency for vegan blacks to screen less than other dietary groups. The blacks in this study, however, were more likely to report screening than the non-blacks. Public health efforts to promote prostate cancer screening among blacks and the fact that this cohort of black Adventist men are relatively well-informed and well-educated, may thus have produced higher compliance and reduced the effect of diet pattern. Others have found that compli- 
ance with prostate cancer screening frequency recommendations among black men can be enhanced by providing support, promoting awareness and the need to take responsibility for sharing correct information and for older men to become role models [57]. These mechanisms may be operating among Adventist black men.

\section{Further studies}

The possible health consequences of avoiding regular prostate cancer screening are still being debated [58]. Thus it is unclear whether the vegans and non-black vegetarians in AHS-2 will suffer any adverse consequences from avoiding prostate cancer screening. It is theoretically plausible that they would be diagnosed at a later stage when clinical symptoms become evident [59], which could potentially lead to lower survival. These questions will therefore be assessed in future studies using new data regarding attitudes and utilization of health and preventive services. However, data from the PLCO study show that even though the 13-year follow-up of men who underwent annual prostate cancer screening reported a relative increase of prostate cancer incidence of $12 \%$ compared to those who were not screened $(R R=1.12$, $95 \% \mathrm{CI}=1.07$ to 1.17 ), there was no survival benefit among those who had been screened [11].

\section{Strengths}

In general, Adventists are health conscious with a wide range of lifestyles [60]. This gives added power when analyzing relationships between lifestyle and various outcomes in healthy groups of the population. The Adventists also enjoy extended life expectancy compared to other groups in the US, specifically connected to lower cancer and cardiovascular disease [61-64]. Another strength is that our study population is recruited from all over the US and Canada, with a large population of both black and non-black men. The study has also collected a large amount of data on numerous lifestyle factors that could be related to both prostate cancer and screening, allowing control for these.

\section{Limitations}

Self-reported screening data has the limitation of errors in recall [65-68]. Federman et al. [69] studied recall bias of PSA screening use, and found that one-third of the patients were unaware that they had PSA test done during their visits/discussion with their physician. Such misclassification is a potential problem in our study as well and could lead to either overestimation or underestimation. There is also the potential for a differential misclassification, for in- stance by race. We have not been able to validate self-report of prostate cancer screening in our study. However, similar associations between diet and compliance with other preventive recommendations (e.g. mammography, colonoscopy) have also been found in AHS-2 data [70]. Finally, attitudes and knowledge about screening, and adequacy of medical insurance were not available in our study.

\section{Conclusion}

Utilization of prostate cancer screening is strongly associated with diet patterns among non-black men and possibly black vegans, but not among other blacks. This difference by race may be due to increased awareness of the higher risk of prostate cancer among the black population. A variety of reasons for the reduced screening rates among vegetarians are discussed, and these await further evidence for better understanding. Further studies are also needed to assess whether this has implications for stage at cancer diagnosis, and survival, especially among vegans.

\section{Abbreviations}

AHS: Adventist Health Study; ACS: American Cancer Society; BMI: body mass index; CI: confidence interval; DRE: digital rectal examination; OR: odds ratio; PSA: prostate specific antigen.

\section{Acknowledgements}

We acknowledge the support of Mrs. Hannelore Bennett, Research Associate, Adventist Health Studies, School of Public Health, Loma Linda University.

\section{Funding/Support}

Project support was obtained from the NIH/National Cancer Institute (1U01CA15293901A1), World Cancer Research Fund, UK (2009/93), and USDA (2010-38938-20924).

\section{Role of the Sponsors}

The funding agencies had no role in the design and conduct of the study; in the collection, management, analysis, and interpretation of data; or in the preparation, review, or approval of the manuscript.

\section{Disclaimer}

The views expressed in this article are those of the authors and do not necessarily represent the views of the NCI or NIFA. The ideas and opinions expressed herein are those of the authors and endorsement by the NCI, NIFA, or their contractors or subcontractors is not intended nor should it be inferred. 


\section{Presentation at Meetings}

These findings were presented by Yermek Ibrayev in a poster session at the Society for Epidemiologic Research annual meeting on June 27, 2012 in Minneapolis, MN.

\section{Ethics Committee Approval and Patient Consent}

This research has been approved by Loma Linda University's review board.

\section{Author Contributions}

Drs. Ibrayev, Fraser, Knutsen had full access to all of the data in the study and take responsibility for the integrity of the data and the accuracy of the data analysis.

Study concept and design: Fraser, Knutsen.

Acquisition of data: Fraser, Knutsen.

Analysis and interpretation of data: Ibrayev,

Oda, Fraser, Knutsen.

Writing, review, revision of the manuscript: Ibrayev, Fraser, Knutsen.

Statistical analysis: Ibrayev, Oda.

Obtained funding: Fraser.

Administrative, technical, or material support: Fraser.

Study supervision: Fraser.

\section{Competing Interests}

Authors had no relevant financial interests.

\section{References}

1. National Cancer Institute UNIoH. Surveillance, Epidemiology, and End Results (SEER) Program; SEER*Stat Database: Table 23.1 Cancer of the Prostate (Invasive); Trends in SEER Incidence and US Mortality Using the Joinpoint Regression Program, 1975-2008 With up to Five Joinpoints, 1992-2008 With up to Three Joinpoints, All Ages by Race/Ethnicity; March 2012. http://seer.cancer.gov/csr/1975_2008/ browse_csr.php?section=23\&page=sect_23_table.01.html.

2. National Cancer Institute UNIoH. Surveillance, Epidemiology, and End Results (SEER) Program; SEER*Stat Database: Table 1.15 Lifetime Risk (Percent) of Being Diagnosed with Cancer by Site and Race/Ethnicity Males, 17 SEER Areas, 2006-2008; Table 1.18 Lifetime Risk (Percent) of Dying from Cancer by Site and Race/Ethnicity Males, Total US, 2007-2009. http://seer.cancer.gov/csr/1975_2008/ browse_csr.php?section $=1 \&$ page $=$ sect_01_table.15.html, http://seer.cancer.gov/csr/1975_2008/browse_csr.php?section=1\&pag e=sect_01_table.18.html.

3. American Society of Cancer. Lifetime Risk of Developing or Dying From Cancer (Information based on SEER 2006-2008 data). 2011.

4. Stavridis S, Saidi S, Lekovski L, Dohcev S, Spasovski G. Screening for prostate cancer: a controversy or fact. Hippokratia. 2010; 14: 170-5.

5. Croswell JM, Ransohoff DF, Kramer BS. Principles of cancer screening: lessons from history and study design issues. Semin Oncol. 2010; 37: 202-15. doi:10.1053/j.seminoncol.2010.05.006.

6. American Cancer Society. American Cancer Society recommendations for prostate cancer early detection; 02/27/2012. 2012.

7. American Urological Association. American Urological Association's Guidelines for prostate cancer screening: 2009 Update. 2009.

8. Ross LE, Taylor YJ, Howard DL. Trends in prostate-specific antigen test use, 2000-2005. Public Health Rep. 2011; 126: 228-39.
9. Oliver SE, May MT, Gunnell D. International trends in prostate-cancer mortality in the "PSA ERA". International journal of cancer Journal international du cancer. 2001; 92: 893-8. doi:10.1002/ijc.1260.

10. Brawley OW. Prostate cancer epidemiology in the United States. World J Urol. 2012; 30: 195-200. doi:10.1007/s00345-012-0824-2.

11. Andriole GL, Crawford ED, Grubb RL, 3rd, Buys SS, Chia D, Church TR, et al. Prostate cancer screening in the randomized Prostate, Lung, Colorectal, and Ovarian Cancer Screening Trial: mortality results after 13 years of follow-up. J Natl Cancer Inst. 2012; 104: 125-32. doi:10.1093/jnci/djr500.

12. National Cancer Institute UNIoH. Surveillance, Epidemiology, and End Results (SEER) Program; SEER*Stat Database:SEER Cancer Statistics Review 1975-2009; Table 23.6; Cancer of the Prostate (Invasive); Age-adjusted U.S. Death Rates by Year, Race and Age. http://seer.cancer.gov/csr/1975_2008/browse_csr.php?section=23\&pa ge=sect_23_table.06.html.

13. McDavid K, Lee J, Fulton JP, Tonita J, Thompson TD. Prostate cancer incidence and mortality rates and trends in the United States and Canada. Public Health Rep. 2004; 119: 174-86.

14. Screening for prostate cancer: U.S. Preventive Services Task Force recommendation statement. Ann Intern Med. 2008; 149: 185-91.

15. Moyer VA. Screening for prostate cancer: U.S. Preventive Services Task Force recommendation statement. Ann Intern Med. 2012; 157: 120-34. doi:10.7326/0003-4819-157-2-201207170-00459.

16. Alexander DD, Mink PJ, Cushing CA, Sceurman B. A review and meta-analysis of prospective studies of red and processed meat intake and prostate cancer. Nutr J. 2010; 9: 50. doi:10.1186/1475-2891-9-50.

17. Steele CB, Miller DS, Maylahn C, Uhler RJ, Baker CT. Knowledge, attitudes, and screening practices among older men regarding prostate cancer. Am J Public Health. 2000; 90: 1595-600.

18. Eisen SA, Waterman B, Skinner CS, Scherrer JF, Romeis JC, Bucholz K, et al. Sociodemographic and health status characteristics with prostate cancer screening in a national cohort of middle-aged male veterans. Urology. 1999; 53: 516-22.

19. Merrill RM. Demographics and health-related factors of men receiving prostate-specific antigen screening in Utah. Prev Med. 2001; 33: 646-52. doi:10.1006/pmed.2001.0940.

20. Moran WP, Cohen SJ, Preisser JS, Wofford JL, Shelton BJ, McClatchey MW. Factors influencing use of the prostate-specific antigen screening test in primary care. The American journal of managed care. 2000; 6: 315-24.

21. Finney Rutten LJ, Meissner HI, Breen N, Vernon SW, Rimer BK. Factors associated with men's use of prostate-specific antigen screening: evidence from Health Information National Trends Survey. Prev Med. 2005; 40: 461-8. doi:10.1016/j.ypmed.2004.07.011.

22. Close DR, Kristal AR, Li S, Patterson RE, White E. Associations of demographic and health-related characteristics with prostate cancer screening in Washington State. Cancer Epidemiol Biomarkers Prev. 1998; 7: 627-30.

23. Beeson WL, Mills PK, Phillips RL, Andress M, Fraser GE. Chronic disease among Seventh-day Adventists, a low-risk group. Rationale, methodology, and description of the population. Cancer. 1989; 64: 570-81.

24. Butler TL, Fraser GE, Beeson WL, Knutsen SF, Herring RP, Chan J, et al. Cohort profile: The Adventist Health Study-2 (AHS-2). International journal of epidemiology. 2008; 37: 260-5. doi:10.1093/ije/dym165.

25. Jaceldo-Siegl K, Fraser GE, Chan J, Franke A, Sabate J. Validation of soy protein estimates from a food-frequency questionnaire with repeated 24-h recalls and isoflavonoid excretion in overnight urine in a Western population with a wide range of soy intakes. Am J Clin Nutr. 2008; 87: 1422-7.

26. [Internet] Codes for States. In: Census Bureau Region and Division Codes and Federal Information Processing System; 1999. http://www.census.gov/popest/geographic/codes02.html.

27. [Internet] Land and freshwater area, by province and territory. In. Statistics Canada; 1999. http://www.statcan.gc.ca/tables-tableaux/ sum-som/101/cst01/phys01-eng.htm.

28. Inc SI. SAS/STAT User's Guide; Version 9.3. Cary, NC: Inc SI. 2012.

29. Tonstad S, Butler T, Yan R, Fraser GE. Type of vegetarian diet, body weight, and prevalence of type 2 diabetes. Diabetes care. 2009; 32: 791-6. doi:10.2337/dc08-1886.

30. Zhu Y, Sorkin JD, Dwyer D, Groves C, Steinberger EK. Predictors of repeated PSA testing among black and white men from the Maryland Cancer Survey, 2006. Prev Chronic Dis. 2011; 8: A114.

31. Swan J, Breen N, Coates RJ, Rimer BK, Lee NC. Progress in cancer screening practices in the United States: results from the 2000 National 
Health Interview Survey. Cancer. 2003; 97: 1528-40. doi:10.1002/cncr.11208.

32. Blackman DK, Bennett EM, Miller DS. Trends in self-reported use of mammograms (1989-1997) and Papanicolaou tests (1991-1997). Behavioral Risk Factor Surveillance System. MMWR CDC surveillance summaries: Morbidity and mortality weekly report CDC surveillance summaries. Centers for Disease Control. 1999; 48: 1-22.

33. Breen N, Kessler L. Changes in the use of screening mammography: evidence from the 1987 and 1990 National Health Interview Surveys. Am J Public Health. 1994; 84: 62-7.

34. Breen N, Wagener DK, Brown ML, Davis WW, Ballard-Barbash R. Progress in cancer screening over a decade: results of cancer screening from the 1987, 1992, and 1998 National Health Interview Surveys. J Natl Cancer Inst. 2001; 93: 1704-13.

35. Martin LM, Calle EE, Wingo PA, Heath CW, Jr. Comparison of mammography and Pap test use from the 1987 and 1992 National Health Interview Surveys: are we closing the gaps? American journal of preventive medicine. 1996; 12: 82-90.

36. Miller BA, Scoppa SM, Feuer EJ. Racial/ethnic patterns in lifetime and age-conditional risk estimates for selected cancers. Cancer. 2006; 106: 670-82. doi:10.1002/cncr.21647.

37. Nadel MR, Blackman DK, Shapiro JA, Seeff LC. Are people being screened for colorectal cancer as recommended? Results from the National Health Interview Survey. Prev Med. 2002; 35: 199-206.

38. Weitzman ER, Zapka J, Estabrook B, Goins KV. Risk and reluctance: understanding impediments to colorectal cancer screening. Prev Med. 2001; 32: 502-13. doi:10.1006/pmed.2001.0838.

39. Zapka JG, Puleo E, Vickers-Lahti M, Luckmann R. Healthcare system factors and colorectal cancer screening. American journal of preventive medicine. 2002; 23: 28-35.

40. Wender RC. Barriers to screening for colorectal cancer. Gastrointestinal endoscopy clinics of North America. 2002; 12: 145-70.

41. Koutros S, Cross AJ, Sandler DP, Hoppin JA, Ma X, Zheng T, et al. Meat and meat mutagens and risk of prostate cancer in the Agricultural Health Study. Cancer Epidemiol Biomarkers Prev. 2008; 17: 80-7. doi:10.1158/1055-9965.EPI-07-0392.

42. Schuurman AG, van den Brandt PA, Dorant E, Goldbohm RA. Animal products, calcium and protein and prostate cancer risk in The Netherlands Cohort Study. Br J Cancer. 1999; 80: 1107-13. doi:10.1038/sj.bjc.6690472.

43. Gallagher RP, Fleshner N. Prostate cancer: 3. Individual risk factors. CMAJ. 1998; 159: 807-13.

44. Walker AR, Walker BF, Tsotetsi NG, Sebitso C, Siwedi D, Walker AJ. Case-control study of prostate cancer in black patients in Soweto, South Africa. Br J Cancer. 1992; 65: 438-41.

45. Mills PK, Beeson WL, Phillips RL, Fraser GE. Cohort study of diet, lifestyle, and prostate cancer in Adventist men. Cancer. 1989; 64: 598-604.

46. Jacobsen BK, Knutsen SF, Fraser GE. Does high soy milk intake reduce prostate cancer incidence? The Adventist Health Study (United States). Cancer Causes Control. 1998; 9: 553-7.

47. Wei MY, Giovannucci EL. Lycopene, Tomato Products, and Prostate Cancer Incidence: A Review and Reassessment in the PSA Screening Era. Journal of oncology. 2012; 2012: 271063. doi:10.1155/2012/271063.

48. Moyad MA, Sakr WA, Hirano D, Miller GJ. Complementary medicine for prostate cancer: effects of soy and fat consumption. Rev Urol. 2001; 3 Suppl 2: S20-30.

49. Chen YC, Chiang CI, Lin RS, Pu YS, Lai MK, Sung FC. Diet, vegetarian food and prostate carcinoma among men in Taiwan. Br J Cancer. 2005; 93: 1057-61. doi:10.1038/sj.bjc.6602809.

50. Adlercreutz H. Phytoestrogens: epidemiology and a possible role in cancer protection. Environ Health Perspect. 1995; 103 Suppl 7: 103-12.

51. Key TJ, Silcocks PB, Davey GK, Appleby PN, Bishop DT. A case-control study of diet and prostate cancer. Br J Cancer. 1997; 76: 678-87.

52. Knutsen SF. Lifestyle and the use of health services. Am J Clin Nutr. 1994; 59: 1171S-5S.

53. Key TJ, Fraser GE, Thorogood M, Appleby PN, Beral V, Reeves G, et al. Mortality in vegetarians and nonvegetarians: detailed findings from a collaborative analysis of 5 prospective studies. Am J Clin Nutr. 1999; 70: 516S-24S.

54. Tonstad S, Stewart K, Oda K, Batech M, Herring RP, Fraser GE. Vegetarian diets and incidence of diabetes in the Adventist Health Study-2. Nutrition, metabolism, and cardiovascular diseases : NMCD. 2011. doi:10.1016/j.numecd.2011.07.004.

55. Tingen MS, Weinrich SP, Boyd MD, Weinrich MC. Prostate cancer screening: predictors of participation. Journal of the American Academy of Nurse Practitioners. 1997; 9: 557-67.
56. Ferrante JM, Shaw EK, Scott JG. Factors influencing men's decisions regarding prostate cancer screening: a qualitative study. Journal of community health. 2011; 36: 839-44. doi:10.1007/s10900-011-9383-5.

57. Woods VD, Montgomery SB, Belliard JC, Ramirez-Johnson J, Wilson $\mathrm{CM}$. Culture, black men, and prostate cancer: what is reality? Cancer Control. 2004; 11: 388-96.

58. Basch E, Oliver TK, Vickers A, Thompson I, Kantoff P, Parnes H, et al. Screening for prostate cancer with prostate-specific antigen testing: American Society of Clinical Oncology Provisional Clinical Opinion. Journal of clinical oncology : official journal of the American Society of Clinical Oncology. 2012; 30: 3020-5. doi:10.1200/JCO.2012.43.3441.

59. Diamandis EP. Prostate cancer screening with prostate-specific antigen testing: more answers or more confusion? Clin Chem. 2010; 56: 345-51. doi:10.1373/clinchem.2009.140046.

60. Montgomery S, Herring P, Yancey A, Beeson L, Butler T, Knutsen S, et al. Comparing self-reported disease outcomes, diet, and lifestyles in a national cohort of black and white Seventh-day Adventists. Prev Chronic Dis. 2007; 4: A62.

61. Tantamango-Bartley Y, Jaceldo-Siegl K, Fan J, Fraser G. Vegetarian Diets and the Incidence of Cancer in a Low-Risk Population. Cancer Epidemiol Biomarkers Prev. 2012; doi:10.1158/1055-9965.EPI-12-1060.

62. Fraser GE. Vegetarian diets: what do we know of their effects on common chronic diseases? Am J Clin Nutr. 2009; 89: 1607S-12S. doi:10.3945/ajcn.2009.26736K.

63. Pettersen BJ, Anousheh R, Fan J, Jaceldo-Siegl K, Fraser GE. Vegetarian diets and blood pressure among white subjects: results from the Adventist Health Study-2 (AHS-2). Public Health Nutr. 2012; 15: 1909-16. doi:10.1017/S1368980011003454.

64. Fraser GE. Diet, life expectancy, and chronic disease : studies of Seventh-Day Adventists and other vegetarians. Oxford ; New York: Oxford University Press; 2003.

65. Howard M, Agarwal G, Lytwyn A. Accuracy of self-reports of Pap and mammography screening compared to medical record: a meta-analysis. Cancer Causes Control. 2009; 20: 1-13. doi:10.1007/s10552-008-9228-4.

66. Rauscher GH, Johnson TP, Cho YI, Walk JA. Accuracy of self-reported cancer-screening histories: a meta-analysis. Cancer Epidemiol Biomarkers Prev. 2008; 17: 748-57. doi:10.1158/1055-9965.EPI-07-2629.

67. Zapka JG. Validation of colorectal cancer screening behaviors. Cancer Epidemiol Biomarkers Prev. 2008; 17: 745-7. doi:10.1158/1055-9965.EPI-08-0179.

68. Abraham L, Geller BM, Yankaskas BC, Bowles EJ, Karliner LS, Taplin $\mathrm{SH}$, et al. Accuracy of self-reported breast cancer among women undergoing mammography. Breast cancer research and treatment. 2009; 118: 583-92. doi:10.1007/s10549-009-0375-4.

69. Federman DG, Goyal S, Kamina A, Peduzzi P, Concato J. Informed consent for PSA screening: does it happen? Effective clinical practice : ECP. 1999; 2: 152-7.

70. Ibrayev Y, Oda K, Dang K, Knutsen SF. Utilization of colorectal cancer screening in persons with different dietary patterns. The Adventist Health Study-2. American journal of epidemiology. 2012; 175 Suppl 11: S29. doi:10.1093/aje/kws258. 